\title{
Un nuevo enfoque en la enseñanza del derecho municipal: seminario de gobierno municipal y seguridad pública
}

\section{A new approach to municipal law education: municipal government seminar and public safety}

\author{
OROZCO-OROZCO, José Zócimo†**
}

Universidad de Guadalajara, Centro Universitario de Ciencias Sociales y Humanidades, División de Estudios Jurídicos. Doctorado en Derecho. Av. Juárez No. 976, Colonia Centro, C.P. 44100, Guadalajara, Jalisco, México.

ID $1^{\mathrm{er}}$ Autor: José Zócimo, Orozco-Orozco

\begin{abstract}
Resumen
La enseñanza del Derecho es dinámica, cada día se incorporan nuevos paradigmas, nuevas leyes, nuevos conocimientos que el profesor y el alumno deben abordar para hacer un correcto análisis de su materia específica. En el caso del Derecho Municipal es preciso incorporar al programa de estudio actual un análisis de Marco Jurídico integral, que forme en el alumno una correcta visión de lo que es su entorno jurídico municipal, interrelacionado con múltiples factores como lo son: la creación de nuevos conceptos, relaciones intermunicipales, regionales, políticas federales, formas de integración, de cooperación en la resolución de conflictos, etc., y que a la vez, lo haga comprender el grado de responsabilidad de las autoridades municipales en la toma de decisiones respecto de su Estado y Nación.
\end{abstract}

Enseñanza, Derecho municipal

\begin{abstract}
Teaching Law should be done in a dynamic way, new concepts are incorporated such as new laws. Students and professors need to use new knowledge to make an analysis of the object of study. In the case of the municipality Law it is important to study the legal framework. The students should study new concepts in intermunicipal cooperation, federal policies, cooperation in conflict resolutions. The responsability of the municipality authorithies when they make desicions should be studied.
\end{abstract}

Citación: OROZCO-OROZCO, José Zócimo. Un nuevo enfoque en la enseñanza del derecho municipal: seminario de gobierno municipal y seguridad pública. Revista de Aplicaciones del Derecho. 2019. 3-8: 13-20.

\footnotetext{
*Correspondencia al Autor (Correo Electrónico: josez_orozco@ hotmail.com)

$\dagger$ Investigador contribuyendo como primer autor.
} 


\section{Introducción}

La enseñanza del Derecho ha utilizado un sistema que se centra en la materia específica, en este caso Derecho Municipal, teniendo un programa rígido, que de alguna manera hace entender a esta materia como algo secundario en el aprendizaje del Derecho, sin vínculo en las materias de importancia, pero que hoy, tenemos a la vista que es imprescindible dominar por su alta interrelación en fenómenos que alteran la pacífica convivencia de nuestra sociedad y que influyen incluso hasta la intervención de los gobiernos estatales y federales en el municipio, como lo es la seguridad pública.

\section{Justificación}

Es la propuesta de una nueva forma de enseñanza del Derecho municipal, basado en seminarios, ejemplos e interrelaciones con otras áreas del Derecho tanto nacional como internacional. Se basa en los cambios que nuestra legislación tiene día con día, en los nuevos puntos de vista que cada vez hacen más importante que el alumno domine una visión íntegra de cada área del conocimiento del Derecho desde las aulas.

Se debe salir de lo tradicional de solo analizar la Constitución Política de los Estados Unidos Mexicanos, en el caso de Jalisco, la Constitución Política del Estado de Jalisco, la Ley del Gobierno y la Administración Pública Municipal del Estado de Jalisco y la Ley Orgánica del Poder Legislativo, para interrelacionarlo en áreas específicas con el Derecho estatal y federal real, no de leyes y decretos, sino de contratos y convenios y políticas públicas que hacen del derecho municipal un micro cosmos, un área tan particular, que su área específica de aplicación, el municipio, se ve impedida de reaccionar ante la avalancha de disposiciones legales que lo comprometen a una nueva dinámica e interrelación con otros municipios, el Estado y la federación.

\section{Elementos metodológicos}

\section{Hipótesis}

Una nueva reingeniería en la enseñanza del Derecho Municipal es necesaria y urgente, dado que cada día se incorporan nuevos paradigmas, nuevas leyes y nuevos conocimientos que el profesor y el alumno deben abordar para hacer un correcto análisis y aplicación del Derecho Municipal, participando activamente en propuestas que beneficien al desarrollo de sus propios municipios.

\section{Planteamiento del problema}

Actualmente, el solo estudio de la Constitución Política de los Estados Unidos Mexicanos, en el caso de Jalisco, la Constitución Política del Estado de Jalisco, la Ley del Gobierno y la Administración Pública Municipal del Estado de Jalisco y la Ley Orgánica del Poder Legislativo es insuficiente para el alumno, que tiene dificultades para integrar este conocimiento a la realidad cotidiana del entorno municipal, sobre todo en determinadas áreas como lo es Gobierno Municipal y la seguridad pública.

\section{Objetivo general}

Mostrar la conveniencia de abordar el conocimiento municipal por medio de módulos o seminarios que como en el ejemplo que se expone: Gobierno Municipal y la seguridad pública, auxilie al alumno a la comprensión íntegra del tema.

\section{Objetivos particulares}

- Aportar una nueva visión sobre la enseñanza del Derecho Municipal, resultado de la experiencia en el aula, también en el área profesional de la impartición de justicia.

- Involucrar al estudiante del Derecho municipal en la lectura y comprensión de leyes nacionales e internacionales como un nuevo método de análisis del entorno municipal, fortaleciendo de esta manera su formación académica. (DIETERICH, Heinz, 2011, p. 262). 
- Actualizar, a través de una nueva metodología, al estudioso del Derecho Municipal en las leyes que de todos los niveles emanen para hacer del municipio el lugar de convivencia ideal de los individuos que en él habitan.

- Resaltar la importancia del municipio en la elaboración de leyes y decretos, de políticas públicas, de contratos y convenios, que le auguren una participación justa y equitativa en el entorno jurídico del Estado y la Nación.

- Que el profesor a través de una exposición de 30 minutos motive la participación del educando en la investigación y ampliación de conclusiones y propuestas en el desarrollo del Derecho Municipal. (SOLIS Solís, Alma Verena, et. al., (2017, p. 76).

\section{Metodología}

Se utiliza el método científico del Derecho, en cuanto a que es un pensamiento generalizador y el método del Derecho municipal, en cuanto a que es un pensamiento individualizador y de ambos, el pensamiento valorizador, de las circunstancias, métodos, leyes y demás ordenamientos jurídicos.

Se emplea el instrumento de la exposición del maestro. (WITKER V., Jorge. 1985, p. 208). Con ayuda de una presentación electrónica que con elementos gráficos, muestren al educando la importancia del Derecho Municipal.

Se emplea el instrumento del ensayo para el alumno, en el afán de concretar los conocimientos adquiridos y completar los esquemas propuestos por el docente.

\section{Importancia de la nueva metodología de enseñanza-aprendizaje del Derecho Municipal}

Actualmente se estudia el siguiente programa:

1) Antecedentes del municipio en México.

2) Marco jurídico del municipio.

3) Disposiciones generales de los municipios, delegaciones y agencias.
4) De los ayuntamientos.

5) De las autoridades municipales.

6) Prevenciones para los casos de ausencia de las autoridades municipales.

7) De la hacienda y patrimonio municipales.

8) De los servicios públicos municipales.

9) De la organización y participación ciudadana vecinal.

10) De la planeación para el desarrollo municipal.

11) De las relaciones del municipio con sus servidores y la seguridad social.

12) Medios de apremio, responsabilidades y de la declaración de situación patrimonial.

13) Actos y procedimientos administrativos.

Se propone una reingeniería en la enseñanza-aprendizaje, en la cual, se tome un papel más activo tanto en la preparación del módulo o seminario por parte del docente y de los educandos, como se muestra en el ejemplo sobre Gobierno Municipal y la seguridad pública, comprendido en el punto 8 del programa actual de la materia.

Salir de la bibliografía municipal, estatal y federal para abordar lo concerniente a los Convenios Internacionales, materia obligada para todo estudioso del Derecho. Salir de las leyes, códigos, decretos, para adentrarse en convenios o contratos con otros gobiernos municipales, estatales o federales. (ROBLES Martínez, Reynaldo. 2009, p. 15).

Ejemplo: conocimientos que el alumno debe adquirir en el proceso de aprender Gobierno Municipal y seguridad pública en Jalisco (UNIVERSIDAD DE GUADALAJARA (1999). Jalisco a futuro, construyendo el porvenir (1999-2025, p. 200).

Nos situamos en el tema de Gobierno Municipal y Seguridad Pública, abordando brevemente el Marco Jurídico de la Seguridad Pública en Jalisco:

- Constitución Política de los Estados Unidos Mexicanos. 
- Convenios Internacionales.

- Constitución Política del Estado de Jalisco.

- Leyes Federales: Ley de Seguridad Nacional, Ley de la Guardia Nacional.

- Leyes Estatales: Ley de Coordinación Metropolitana del Estado de Jalisco, Ley del Sistema de Seguridad Pública para el Estado de Jalisco.

Comentario: Si bien, las leyes federales y estatales conforman un universo más amplio, será tarea del educando completar el esquema y verificarlo con su maestro a través de algún ensayo posterior.

\section{Constitución Política de Los Estados Unidos Mexicanos, Artículo 16}

Cualquier persona puede detener al indiciado en el momento en que esté cometiendo un delito e inmediatamente después de haberlo cometido, poniéndolo sin demora a disposición de la autoridad civil más cercana y ésta con la misma prontitud, a la del Ministerio Público. Existirá un registro inmediato de la detención.

Solo en casos urgentes, cuando se trate de delito grave así calificado por la ley y ante el riesgo fundado de que el indiciado pueda sustraerse a la acción de la justicia, siempre y cuando no se pueda ocurrir ante la autoridad judicial por razón de la hora, lugar o circunstancia, el Ministerio Público podrá, bajo su responsabilidad, ordenar su detención, fundando y expresando los indicios que motiven su proceder.

En casos de urgencia o flagrancia, el juez que reciba la consignación del detenido deberá inmediatamente ratificar la detención o decretar la libertad con las reservas de ley.

\section{Convenciones Internacionales}

Entre otras son importantes por su aplicación, las siguientes:

- Declaración Universal de los Derechos Humanos.

- Pacto Internacional sobre Derechos Civiles y Políticos.
-Convención Americana sobre Derechos Humanos.

- Convención Interamericana para Prevenir y Sancionar la Tortura.

- Convención Interamericana sobre Desaparición Forzada de Personas.

- Convención Interamericana para el cumplimiento de Condenas Penales en el Extranjero.

- Declaración sobre los Principios Fundamentales de Justicia para las Víctimas de Delito y Abuso de Poder. Adoptada por la Asamblea General de las Naciones Unidas.

Principios básicos sobre el empleo de la fuerza y de armas de fuego por los funcionarios encargados de hacer cumplir la Ley adoptados por el Congreso de las Naciones Unidas.

Comentario: El docente además de enunciar, explicará brevemente la trascendencia de dichas convenciones internacionales.

\section{Ley De Seguridad Nacional. Artículo 5}

Art. 5. Para efectos de la presente Ley, son amenazas a la Seguridad Nacional:

I. Actos tendientes a consumar espionaje, sabotaje, terrorismo, rebelión, traición a la patria, genocidio, en contra de los Estados Unidos Mexicanos dentro del territorio nacional.

III. Actos que impidan a las autoridades actuar contra la delincuencia organizada.

IV. Actos en contra de la seguridad de la aviación.

V. Actos que atenten en contra del personal diplomático.

XI. Actos tendientes a obstaculizar o bloquear actividades de inteligencia o contrainteligencia.

XII. Actos tendientes a destruir o inhabilitar la infraestructura de carácter estratégico o indispensable para la provisión de bienes o servicios públicos. 


\section{Ley de la Guardia Nacional}

Objeto de la Guardia Nacional:

Artículo 5. El objeto de la Guardia Nacional es realizar la función de seguridad pública a cargo de la Federación y, en su caso, conforme a los Convenios que para tal efecto se celebren: Colaborar temporalmente en las tareas de Seguridad Pública que corresponden a la Entidades Federativas o Municipios".

--Fines de la Guardia Nacional: Nacional:

Art. 6. Son fines de la Guardia

...IV. Llevar a cabo acciones de colaboración y coordinación con entidades federativas y municipios.

Art. 7. Para materializar sus fines, la Guardia Nacional deberá:

...IV. Colaborar en materia de Seguridad Pública, con las Entidades Federativas y Municipios, en los términos que así se convenga, de conformidad con las disposiciones que regulen el Sistema Nacional de Seguridad Pública.

Comentario: El docente, además de referir estas leyes, sus fines y objetivos, pondrá énfasis en su aplicación al interior del municipio.

\section{--Constitución Política Del Estado De Jalisco. Artículos 8 y 53}

Art. 8. La investigación de los delitos corresponde al Ministerio Público y a las Policías, las cuales actuarán bajo conducción y mando de aquel en el ejercicio de esta función...

Art. 53. La investigación de los delitos del fuero común y concurrente, y la persecución ante los tribunales de los responsables en su comisión, corresponden al Ministerio Público a cargo del Fiscal General, quien se auxiliará de las policías, las que estarán bajo su mando inmediato, en los términos del Artículo 21 de la Constitución Política de los Estados Unidos Mexicanos.
Comentario: El docente hará mención de otros artículos en referencia al municipio, pero en esta materia de Seguridad pública dará énfasis a los artículos 8vo. y 53 avo.

\section{--Ley De Coordinación Metropolitana Del Estado De Jalisco. Arts. 3, 7, 15, 20, 23, 37}

Art. 3. Área Metropolitana es el centro de población, geográficamente delimitado, asentado en el territorio de dos o más municipios, con una población de cuando menos cincuenta mil habitantes, declarado oficialmente con ese carácter por decreto del Congreso del Estado; o en su caso, la reconocida como Zona Metropolitana administrativamente por los procedimientos $\mathrm{y}$ leyes aplicables.

Art. 7: Requisitos de procedencia de Región Metropolitana: Para que proceda la declaración de una Región Metropolitana se requiere:

--Que se integre por un área metropolitana constituida previamente y uno o más centros de población ubicados fuera de la delimitación geográfica de aquella, dentro del Estado, con los que exista cercanía geográfica y tendencias de crecimiento que los acerquen;

--Que el titular del Poder Ejecutivo del Estado proponga su declaración oficial ante el Congreso del Estado, y

--Que se demuestre la existencia de relaciones socioeconómicas entre los municipios que constituirán una Región Metropolitana.

Art. 15. Efectos de La Declaratoria de Área o Región Metropolitana

I. El reconocimiento oficial como Área o Región Metropolitana.

II. La autorización al titular del Poder Ejecutivo del Estado para participar en la integración de la instancia de coordinación política de área o región metropolitana, en representación del Estado o a través de un representante. 
III. La autorización al titular del Poder Ejecutivo del Estado para destinar, conjuntamente con los municipios, recursos financieros, humanos y materiales estatales para la organización y funcionamiento de la instancia de planeación metropolitana, en los términos del Presupuesto de Egresos correspondiente y de las demás disposiciones aplicables.

Art. 20. Del interés público: Son materias de interés público, para efectos de Coordinación y Asociación metropolitanas, las siguientes:

XIII. La seguridad pública.

Art. 23. Revisión y modificación del convenio. Para incluir a un municipio en Convenio de Coordinación Metropolitana se requiere que:

I. El municipio interesado o parte del mismo, forme parte del Área o Región metropolitana en los términos de la declaración oficial correspondiente expedida por el Congreso del Estado.

Art. 37. De los fines de las Agencias Metropolitanas.

1. Las Agencias Metropolitanas son las instancias de establecer los objetivos y estrategias y llevar a cabo acciones en una de las materias de la Agenda Metropolitana, que por su complejidad, requiere de un organismo enfocado a ella específicamente.

\section{--Ley del Sistema de Seguridad Pública para EI Estado De Jalisco, Arts. 14, 26 *}

Art. 14. Las autoridades competentes en materia de Seguridad Pública del Estado y los municipios se coordinarán para:

\section{Integrar el Sistema Estatal de} Seguridad Pública

II. Determinar las políticas de seguridad pública, prevención del delito, así como ejecutar, dar seguimiento y evaluar sus acciones, a través de las instancias previstas en esta Ley.
VII. En los municipios del Estado se podrán formalizar los Convenios de Colaboración respectivos para brindar los servicios de seguridad pública que se estimen pertinentes bajo la coordinación del Titular del Poder Ejecutivo del Estado.

Art. 26. Son cuerpos de seguridad pública de Jalisco:

IV. Los cuerpos de seguridad pública municipales, con todas las unidades y agrupamientos que señale su reglamento;

V. Los demás que en el futuro se constituyan con estricto respeto de esta Ley.

--Objetivo de la nueva Policía Metropolitana del Estado De Jalisco.

Creación de la elaboración del modelo operativo policial para detectar las amenazas y delitos, para fortalecer y coordinar las policías de los Ayuntamientos Metropolitanos, para Optimizar esfuerzos y recursos y para articular con las fuerzas federales y reducir y afrontar la delincuencia.

Comentario: En relación a la materia de estudio sobre seguridad pública se podrá enlazar el contenido con la nueva visión del gobierno respecto de la Refundación en Jalisco.

--Creación en Jalisco de estructura que de acuerdo a derechos humanos garantice el acceso al derecho de la verdad que ostentan las víctimas y la sociedad:

- Unidad de Transparencia de la Fiscalía

- Sistema de Información sobre Víctimas de Desaparición en Jalisco (SISOVID).

Comentario: En éstos encontramos que se realizan y difunden estadísticas sobre actos de violencia y enfrentamientos, tratando de informar con veracidad de los ilícitos cometidos en Jalisco. Ees pertinente enlazar con la labor de la Sociedad civil, que en conjunto con la estructura gubernamental y de acuerdo a convenciones internacionales trabajan en el perfeccionamiento de estos datos estadísticos. 


\section{--Participación de la sociedad civil}

Propuestas del Centro de Justicia para la Paz y el Desarrollo (CEPAD).

La Sociedad Civil de Jalisco ha solicitado específicamente los siguientes puntos:

- Desglose de información específica sobre el delito por municipio, para conocer la situación de violencia y riesgo en la comunidad al interior del Estado.

- Enfoque diferenciado (menores de edad, mujeres, extranjeros, defensores de derechos humanos y periodistas, etc. Como lo establece la Ley General en materia de desaparición forzada de personas, cometida por particulares y del Sistema Nacional de Búsqueda.

- Perfiles de las víctimas.

- Patrones de desaparición y modus operandi.

- Información geográfica y geolocalización sobre las zonas con mayor incidencia del delito.

- Información detallada sobre la situación de los municipios y ciudades al interior del Estado.

- Eficacia de las estrategias de búsqueda e investigación, número de personas reportadas como desaparecidas, a) con vida, b) sin vida.

\section{Conclusiones}

Primero: La enseñanza del módulo de derecho Municipal, debe comprender una exposición breve del maestro que incluya la visión integral de la Seguridad Pública en Jalisco y sus municipios, lo que auxiliará al educando a formar una concepción real del problema y las soluciones que a partir de esferas públicas y privadas, nacionales e internacionales, se aplican en el entorno municipal.

Segundo: El educando participará en propuestas de cambio de legislación interna en su municipio, al contar con elementos que le auxilien a analizar con veracidad los elementos de cambio reales en los nuevos paradigmas de seguridad pública internacional, nacional, estatal y municipal.
Tercero: Esta nueva forma dinámica de enseñanza del Derecho contribuye a dotar al educando de una visión global de aplicación del Derecho municipal y a dejar la apatía que se forma cuando se considera que no tiene consecuencias mayores dicha enseñanza, al conocer aparte de la bibliografía formal del programa, convenios tanto internacionales, nacionales, estatales e intermunicipales que influyen en el desempeño del mismo municipio.

Cuarto: El Derecho Municipal en esta nueva concepción, deja de tener el status de materia opcional, para convertirse en materia del tronco esencial del aprendizaje del abogado.

Quinto: El Derecho Municipal en el sistema semiescolarizado de la carrera de Licenciado en Derecho de la Universidad de Guadalajara, es estudiado por alumnos de los últimos semestres de la Licenciatura, motivo por el cual se propone que sea su enseñanza de tipo seminario e incluso en un auditorio o sala dotado de proyector, pizarrón interactivo, etc., para facilitar la exposición del docente y la participación de los educandos en sesiones de no más de 50 minutos por tema.

Sexto: La nueva forma de enseñar el Derecho Municipal proporciona la oportunidad al educando de ir más allá de solo pasar la materia para obtener unos créditos. Es la oportunidad de hacer investigación, tanto bibliográfica como de campo, en su municipio de interés, aportar soluciones para su entorno, vertido este conocimiento en un ensayo que podrá convertirse en artículos, revistas o libros temáticos bajo la coordinación de personas expertas del tema.

Se propone además como parte de la evaluación, un examen de conocimientos que comprenda el módulo de enseñanza respectivo y acudir a una sesión de Ayuntamiento para hacer un informe de los aspectos tratados en la actividad municipal presenciada y su fundamento legal.

\section{Referencias}

Constitución Política de los Estados Unidos Mexicanos.

Constitución Política del Estado de Jalisco.

Convenios Internacionales. 
DIETERICH, Heinz. (2011). Nueva guía para la investigación científica, Orfila.

Ley de Coordinación Metropolitana del Estado de Jalisco.

Ley de la Guardia Nacional.

Ley de Seguridad Nacional.

Ley del Sistema de Seguridad Pública para el Estado de Jalisco.

ROBLES Martínez, Reynaldo. (2009). El Municipio, Porrúa.

SOLIS Solís, Alma Verena, et. al., (2017). "Prácticas y elementos requeridos en ambientes de aprendizaje universitario", en Revista de Educación Básica, Ecorfán Perú, Dic. 2017. Vol. 1. No. 2.

TAYLOR, S.J., BOGDAN, R. (1994). Introducción a los métodos cualitativos de investigación, Paidós.

UNIVERSIDAD DE GUADALAJARA (1999). Jalisco a futuro, construyendo el porvenir (1999-2025).

WITKER V., Jorge. (1985), Técnicas de la enseñanza del Derecho, PAC/UNAM. 